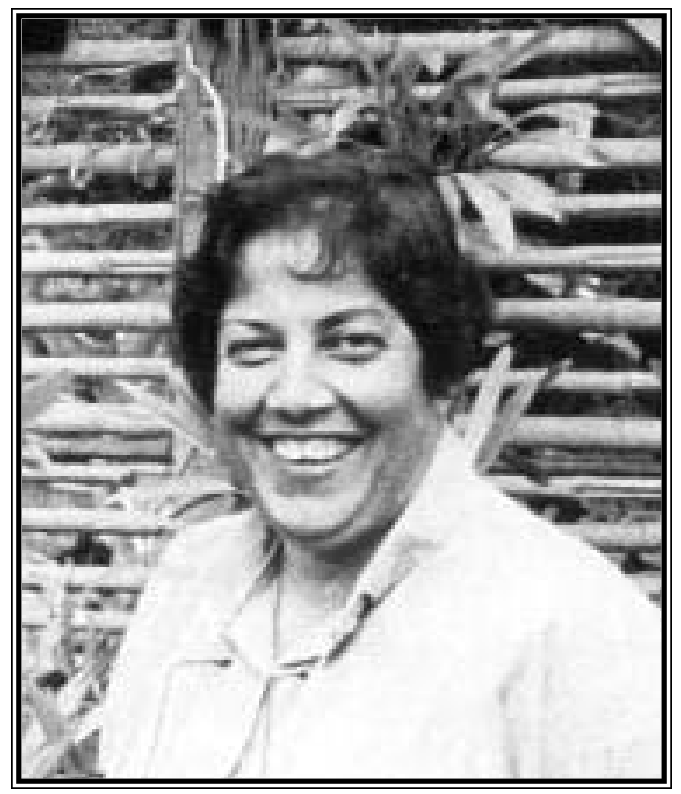

Dora Emilia Mora de Retana

(1940-2001) 


\title{
DORA EMILIA MORA DE RETANA, 1940-2001
}

\author{
Carlos O. Morales \\ Jardín Botánico Lankester, Apdo. 1031-7050 Cartago, Costa Rica, y \\ Escuela de Biología, Universidad de Costa Rica. 2060 San José, Costa Rica
}

El 12 de julio de 2001 murió Dora E. Mora de Retana, quien desde 1979 hasta febrero de 2000 fue directora del Jardín Botánico Lankester, de la Universidad de Costa Rica (U.C.R.). Ella dedicó la mayor parte de su energía vital a estudiar las orquídeas y a promover la conservación de la flora tropical; esto último desde su nombramiento como autoridad científica en el Comité de Flora de la Convención Internacional sobre Comercio de Especies Amenazadas (CITES). Después de Rafael Lucas Rodríguez (1915-1981), ella fue durante mucho tiempo la única orquideóloga costarricense con formación académica y científica en el campo de la botánica.

Dora Emilia nació el 24 de agosto de 1940 en Cartago. Realizó los estudios primarios en la Escuela Sagrado Corazón de Jesús, los secundarios en el colegio homónimo. En la Escuela de Biología (U.C.R.) obtuvo los títulos de Profesora en Ciencias, Bachiller en Biología y Licenciada en Biología. En 1969 empezó a laborar en la Cátedra de Fundamentos de Biología, Escuela de Estudios Generales (U.C.R.). En 1973 inició labores en la Cátedra de Biología General de la Escuela de Biología. En esta misma unidad de la U.C.R. empezó en 1978 con la Cátedra de Botánica General y a partir de 1984 ofreció el curso de Orquideología, entre otros. Los primeros estudios publicados por Dora Emilia (ver adelante) se referían a la anatomía y al desarrollo de una especie muy conocida por los orquideólogos y aficionados a las orquídeas en Costa Rica: Acnistus arborescens (L.) Schltdl. (Solanaceae), un arbusto o árbol pequeño llamado güitite, a cuya corteza suave y corchosa se adhieren fácilmente las raíces de las orquídeas epífitas.

Con la dirección de Dora Emilia, lenta pero progresivamente el Jardín Botánico Lankester dejó de ser una pequeña finca de 10,7 hectáreas para convertirse en un jardín botánico de renombre mundial, especialmente por su extraordinaria colección de orquídeas. Gran parte del desarrollo y del progreso del Jardín se deben al esfuerzo y a la energía de Dora Emilia y al empeño de los buenos trabajadores que siempre ha tenido el Jardín. La capacidad de trabajo de Dora Emilia muchas veces me sorprendió: podía empezar su día de trabajo a las 7 horas y estar activa sin descanso hasta las 20 horas, todavía en los últimos años cuando ya podía haberse pensionado y retirado. Sus actividades eran tan versátiles, que en un solo día podía visitar el Jardín Lankester, en Cartago, para coordinar labores prioritarias, ir a San José a dar clases de botánica u orquideología, viajar al Ministerio de Recursos Naturales (ahora, Ministerio del Ambiente) a atender algún asunto propio de CITES, ir a tocar las puertas de las autoridades universitarias para exigirles apoyo para el Jardín Lankester y, no pocas veces, al final del día, visitar a Joaquín García ${ }^{1}$ para identificar especies y, juntos, preparar publicaciones sobre orquídeas. Sin embargo, una vez Dora Emilia me reveló que se cansaba mucho trabajando en herbarios; fue durante una de las visitas de John T. Atwood (SEL) al Herbario de la Universidad de Costa Rica (USJ) y al Herbario Nacional de Costa Rica (CR). "Él, en cambio, es incansable", me dijo Dora Emilia en CR señalando a Atwood, quien estaba de pie y muy concentrado revisando ejemplares de Maxillaria. Es obvio que Dora Emilia prefería estudiar sus queridas orquídeas in vivo.

Muchas personas recuerdan a Dora Emilia como una persona accesible, comunicativa, enérgica, dis-

${ }^{1}$ El Dr. Joaquín B. García falleció el 22 de agosto del 2001 y sus cenizas yacen desde el 2 de septiembre en el Jardín Botánico Lankester. En dos meses consecutivos, Costa Rica perdió a sus dos mejores orquideólogos nativos. 
puesta siempre a ayudar y a compartir ideas y conocimientos. Así la recuerdo yo desde que fui su asistente en el Jardín Botánico Lankester y cuando, por no haber otros estudiantes interesados, tomé su curso de Orquideología por tutoría. De este modo el trato era mucho más personal que en un grupo de estudiantes. Yo la seguía por el Jardín Lankester, anotando en mi cuaderno las características de géneros y especies que ella me mostraba. Recuerdo muy bien que jamás perdió la paciencia conmigo; todo lo contrario: parecía muy satisfecha de que alguien más se interesara por las orquídeas y era evidente que le gustaba transmitir sus conocimientos sobre estas plantas. Yo sé muy bien que otros no piensan igual. El carácter generalmente sobrio y apacible de Dora Emilia se volvía férreo e irreductible al defender sus ideas y sus acciones frente a las de otros; una virtud que muchos costarricenses no tienen, todavía fuertemente influidos por preceptos (pseudo)reli-giosos que dan a entender que oponerse a los argumentos de otros sólo genera rencor. Esto no es nuevo; ya lo habían notado los antiguos romanos: "obsequium amicos, veritas odium parit"; es decir, la cortesía hace amigos, mientras que la verdad genera odio. Sin embargo, especialmente en el ámbito científico y cuando se trata de hacer progresar a una sociedad que, en varios aspectos, está anquilosada, es indispensable la confrontación de ideas y la discusión nudis verbis, para lograr el mejor acuerdo.

La huella de Dora Emilia y sus contribuciones a la orquideología perdurarán sobre todo en el Jardín Botánico Lankester, en el Herbario de la Universidad de Costa Rica (USJ) y en sus publicaciones, donde se preserva su legado científico y el fruto de su esfuerzo práctico.

\section{ESPECIES DESCRITAS POR DORA EMILIA MORA DE RETANA, COMBINACIONES NUEVAS Y NOMBRES INÉDITOS}

En total, son 10 especies nuevas, 7 combinaciones nuevas, 4 táxones inéditos y 3 nombres de herbario. Todos los ejemplares de los herbarios USJ y CR, citados a continuación, fueron recolectados en Costa Rica.

Cyclopogon prasophylloides (Garay) Mora-Ret. \& J. García, Brenesia 37: 80. 1992.

Basiónimo: Beadlea prasophylloides Garay, Bot. Mus. Leafl. 28: 301. 1982.

Con excepción de C. olivaceus (Rolfe) Schltr., tanto en CR como en USJ los ejemplares de este género terrestre están sin determinar, lo que parece reflejar la dificultad de estudiarlo.

Encyclia ionocentra (Rchb.f.) Mora-Ret. \& J. García, Brenesia 33: 124. 1990.

Basiónimo: Epidendrum ionocentrum Rchb.f., Gard. Chron. N.s. 20: 8. 1883.

Epidendrum prismatocarpum Rchb.f. var. iono centrum (Rchb.f.) Teuscher, Amer. Orch. Soc. Bull. 38: 398. 1969.

Hormidium ionocentrum (Rchb.f.) Brieg, Orchidee (Hamburg) 3. Auf. 1: 576. 1977.

Prosthechea ionocentra (Rchb. f.) W.E. Higgins, Phytologia 82(5): 378. 1997.

La publicación en Brenesia citada arriba incluye una comparación descriptiva y dibujos de las flores de Encyclia ionocentra y E. prismatocarpa (Rchb.f.) Dressler; la primera ha sido confundida con la segunda. Siguiendo a Higgins en Phytologia, ambas especies se tratan ahora en el género Prosthechea, como:

Prosthechea ionocentra (vide supra), Puntarenas; Monteverde, Bullpen, ca. 1550 m. F. Pupulin, Dora E. Mora, G. Barbosa \& curso de Orquideología \# 880. 16/7/1998 (USJ-71146) y

Prosthechea prismatocarpa (Rchb.f.) W.E. Higgins, San José; Pérez Zeledón, Villa Mills, Carretera Interamericana, Cordillera de Talamanca, ca. 3000 m. Gustavo Vargas \# 302. 26/4/1987 (USJ-71202).

"Encyclia tardiflora (sp. nova?)", nom. herb.

Este nombre inédito ha sido utilizado por R. L. Dressler y Dora E. Mora para un taxon del cual existen dos ejemplares en USJ:

Alajuela; Palmares, orillas del Río Rastrojos. "Florece en pseudobulbos viejos" [fide Dora E. Mora, de allí el nombre propuesto "tardiflora"]. Wilber Barahona s.n., en floración el 26/II/1994 (USJ-51074).

Alajuela; Palmares, a orillas del Río Jesús María. Dora E. Mora s.n. Marzo de 1994 (USJ-57851). 
La especie referida debe tratarse actualmente en el género Prosthechea.

"Epidendrum boothii (S.) Mora \& García", nom. herb.

Este nombre, así anotado en un ejemplar de herbario: Heredia; Horquetas de Sarapiquí, Quebrada El Tigre. 600 m. Tania Bermúdez \# 5. 14/6/1997 (USJ-63874), es un error, porque existe Epidendrum boothii (Lindl.) L.O. Williams, Ann. Missouri Bot. Gard. 26(4): 282. 1939, cuyo basiónimo es Maxillaria boothii Lindl., Edwards's Bot. Reg. 24: misc. 52-53. 1838.

En todo caso, en mayo de 2001 Éric Hágsater anotó en el ejemplar el nombre de la especie en uso actualmente: Nidema boothii (Lindl.) Schltr.

Epidendrum crassus [sic] (Dressler) Mora-Ret. \& J. García, Brenesia 33: 124. 1990.

Basiónimo: Epidanthus crassus Dressler, Orquídea (Méx.) 9(1):15. 1983.

Sin embargo, ya existía Epidendrum crassum C. Schweinf., Bot. Mus. Leafl. 15: 141. 1952. Un principio básico del Código Internacional de Nomenclatura Botánica es que dos especies diferentes no pueden tener el mismo nombre científico. Así, Barringer propuso el nuevo nombre Epidendrum insolatum Barringer, Brittonia 42(4): 245. 1991 [1992] para el taxon descrito por Dressler como Epidanthus crassus.

En Brenesia 37: 126. 1992, Mora-Retana \& J. García mencionan "Epidendrum incrassatum", como un nombre sugerido por Robert Dressler para Epidanthus crassus. Sin embargo, desafortunadamente el artículo se publicó incompleto (¡faltó una página!), de modo que ese nombre nunca se publicó válidamente. Cuando me di cuenta de este error en Brenesia, le sugerí a Dora Emilia que publicara la información faltante en el siguiente número de la revista; no obstante, ella ya no tenía voluntad para hacerlo, porque tenía sus energías concentradas en nuevas publicaciones y proyectos. De modo que el nombre asignado por Barringer a esta especie tiene prioridad.

Cartago; Cordillera de Talamanca, km 37, Carretera Interamericana sur. Dora E. Mora \& Rosa Sandoval s.n. Abril de 1990 (USJ-37187, 37188).
Epidendrum epidendroides (Garay) Mora-Ret. \& J. García, Brenesia 33: 124. 1990.

Basiónimo: Neowilliamsia epidendroides Garay, Orch. Dig. 41(1): 20-21. 1977.

Cartago; Parque Nacional Tapantí, sendero Palmito, 1450 m. Dora E. Mora s.n. 22/10/1992 (USJ-45209).

Epidendrum talamancanum (J.T. Atwood) MoraRet. \& J. García, Brenesia 33: 124. 1990.

Basiónimo: Epidanthus talamancanus J.T. Atwood, Icon. Pl. Trop. 14, pars 1: pl. 1315. 1989.

Cartago/San José; Cordillera de Talamanca, km 36, Carretera Interamericana sur, $1850 \mathrm{~m}$. Inge Mack \# 15 (USJ-49873).

Varios nombres genéricos utilizados en décadas pasadas se tratan actualmente como sinónimos de Epidendrum L. Entre éstos, tenemos en la flora de Costa Rica Epidanthus L.O. Williams, Nanodes Lindl., Neolehmannia Krnzl., Neowilliamsia Garay y Physinga Lindl. Los estudios epidendrológicos llevados a cabo por Éric Hágsater (AMO) han sido críticos para entender poco a poco la circunscripción del género Epidendrum y poder diferenciarlo de los géneros vecinos, como Encyclia y Prosthechea.

Fernandezia tica Mora-Ret. \& J. García, Brenesia 39-40: 164. 1993.

El 27 de octubre de 1992 fue sorprendente el hallazgo repentino de una sola planta florecida de esta especie en la zona de Vara Blanca, Heredia, a ca. $2000 \mathrm{~m}$ de elevación. La única evidencia anterior de esta especie era una acuarela pintada por Rafael Lucas Rodríguez en agosto de 1977, de "entre El Empalme y Ojo de Agua", Cordillera de Talamanca (vide Rodríguez, R.L. et al. 1986. Géneros de orquídeas de Costa Rica. San José, Edit. Univ. de Costa Rica. p. 253). En la acuarela se anotó Fernandezia costaricensis (Ames \& C. Schweinf.) Garay \& Dunsterv., un nombre que no debe usarse por choque de sinonimia con otra especie diferente: Pachyphyllum costaricense (Ames \& Schweinf.) L.O. Williams. Por ello fue necesario tanto asignar un nuevo nombre como 
describir y publicar la nueva especie. En 1997 fueron halladas otras plantas de esta especie.

Heredia; camino a Vara Blanca, ca. 2000 m. John T. Atwood \& Óscar Rodríguez \# 4181. 27/10/1992 (Holotipo, USJ-45216).

Heredia; Cerro Chompipe, 2200 m. Dora E. Mora \& R. L. Dressler s.n. 27/8/1997 (USJ-67288).

Kefersteinia excentrica Dressler \& Mora-Ret., Orquídea (Méx.) 13(1-2): 261. 1993.

Cartago; La Selva, camino a Taus, 1300-1400 m. R.L. Dressler \& Dora E. Mora s.n. 9/11/1984 (Holotipo, USJ-45426, isotipo en alcohol, USJ).

Alajuela; Cataratas de San Ramón. Dora E. Mora s.n. Julio de 1990 (USJ-45427, indicado como paratipo, con un duplicado en alcohol).

Macroclinium doderoi Mora-Ret. \& Pupulin, Selbyana 18(1): 7. 1997.

Cartago; Turrialba, Caño Seco. Torito, ca. $1500 \mathrm{~m}$.

Robert L. Dressler, Dora E. Mora \& R. Dodero s.n. 19/7/1994 (Holotipo, USJ-57025).

Macroclinium robustum Pupulin \& Mora-Ret., Selbyana 18(1): 7 \& 10. 1997.

San José; Alajuelita, en lomas sobre Barrio La

Mora, ca. 1850 m. Franco Pupulin \& M. Flores \# 315. 17/7/1995 (Holotipo, USJ-57743, isotipo, USJ-68694).

Maxillaria cedralensis J.T. Atwood \& Mora-Ret., Selbyana 18(1): 31.1997

San José; Cordillera de Talamanca, Dota, El Cedral. 2000 m. Dora E. Mora \& curso de Orquideología s.n. Abril de 1990. (Holotipo, USJ-31579).

"Maxillaria humilis Atwood \& Mora-Retana, ined.”, Brenesia 37: 99. 1992.

Nombre inédito incluido en la lista de orquídeas de Costa Rica.

Maxillaria rodrigueziana J.T. Atwood \& MoraRet., Icon. Pl. Trop. 14: pl. 1361. 1989.

Esta especie fue dibujada por Rafael Lucas Rodríguez en junio de 1974 (vide Rodríguez, R.L. et al. 1986. Géneros de Orquídeas de Costa Rica.
San José, Edit. Univ. de Costa Rica. p. 215) y fue dedicada a él póstumamente. Había sido confundida con Maxillaria speciosa Rchb.f. de Sudamérica y con M. arachnitiflora Ames \& Schweinf. El tipo de $M$. rodrigueziana es de El Cope, Panamá ( $T$. Antonio 2137, MO, SEL), pero la especie se halla también en Costa Rica a alturas medias de la Cordillera de Talamanca. Es una de las especies más atractivas del género en Costa Rica y sus flores (entre junio y julio) son muy fragantes.

San José; La Paulina, San Pedro, Montes de Oca. 1160 m. Planta cultivada. Rafael Lucas Rodríguez \#1003A. 5/6/1965 (2 flores) (USJ10937).

“Oerstedella satanica Hágsater \& Mora-Retana, ined.", Brenesia 37: 101. 1992.

Así se menciona en la lista de orquídeas, pero permanece inédita. En USJ se usó provisionalmente un segundo nombre para el mismo taxon: "Oerstedella diabola, ined.". El Ing. Éric Hágsater (AMO) me comunicó en julio de 2001 que todavía no está seguro de la identidad de este taxon, por cuanto hay varias especies similares involucradas. El epíteto satánica se refiere al callo del labelo "con dos proyecciones elevadas semejantes a cuernos", según la descripción inédita.

San José; Cordillera de Talamanca, Pérez Zeledón, Villa Mills, ca. 3100 m. Dora E. Mora s.n. Marzo de 1990 (USJ-31992, 32890, 32889).

Ornithocephalus grex-anserinus Dressler \& MoraRet., in Dressler, Novon 7(2): 120. 1997.

Esta extraña y bella planta ha sido recolectada solamente una vez. El aspecto de aves en vuelo de las flores motivó a los autores para asignar el nombre latino grex anserinus (bandada de gansos).

Alajuela; cantón Alajuela, distrito Sarapiquí, alrededor de la Laguna María Aguilar. Dora E. Mora, F. Pupulin \& A. Herrera s.n. 5/5/1992, en floración el 11/11/1993 en el Jardín Botánico Lankester, cuando preparé el espécimen tipo (Holotipo: USJ-49975).

Reichenbachanthus lankesteri (Ames) Mora-Ret. \& J. García, Brenesia 37: 81. 1992. 
Basiónimo: Hexisea lankesteri Ames, Sched. Orch. 9: 47. 1925.

Sin embargo, existe un basiónimo anterior y, por tanto, prioritario, que había pasado desapercibido durante mucho tiempo; se trata de Scaphyglottis subulata Schltr., del cual Dressler hizo la combinación válida ahora para esta especie: Reichenbachanthus subulatus (Schltr.) Dressler.

No existe hasta ahora material de esta especie en USJ. En CR sólo un ejemplar, determinado hace muchos años por C. Schweinfurth como Hexisea lankesteri: Alajuela; San Ramón. La Palma. 1175 m. Alberto M. Brenes 2292. 29/8/1924 (CR-33931).

Scaphyglottis geminata Dressler \& Mora-Ret., Orquídea (Méx.) 13(1-2): 192. 1993.

Cartago; Turrialba, Pejibaye, Taus. Dora E. Mora \& R.L. Dressler \# 31. 20/8/1984 (USJ-32348). [En vez de agosto, podría ser octubre, al comparar con otro espécimen de Dressler].

Trichocentrum costaricense Mora-Ret. \& Pupulin, Selbyana 15(2): 94. 1994.

Alajuela; San Ramón, Los Lagos. 800 m. Mario Blanco 1813, Robbin Moran, E. Watkins, E. Vargas. 23 de enero de 2001 (USJ-76755).

Trichocentrum dianthum Pupulin \& Mora-Ret., Selbyana 15(2): 90 \& 92. 1994.

San José; Pérez Zeledón, Las Nubes de Quizarrá, 1000 m. J. Cambronero s.n. 1988 (Erbario Franco Pupulin). Floreció en cultivo en marzo de 1989 (Holotipo, USJ-38972).

“Trichopilia pendula J. García \& Mora-Retana, ined.”, Brenesia 37: 113. 1992.

Nombre anotado en la lista de orquídeas, pero no se publicó.

“Trichopilia x ramonensis J. García \& MoraRetana, ined. (nat. hyb.)”, Brenesia 37: 113. 1992.
Nombre incluido en la lista de orquídeas para designar un híbrido natural: T. marginata Henfr. x T. suavis Lindl. En Die Orchidee 51(4): 443-445, Ehrenfried Lucke publicó notas sobre reproducción y floración de este híbrido. El naturalista alemán Clarence Klaus Horich (1930-1994) había enviado semillas a Alemania en enero de 1993, que exitosamente dieron origen a plantas que florecieron siete años después. Lucke señala, de paso, que el nombre Trichopilia $\mathrm{x}$ ramonensis no ha sido válidamente publicado, lo cual es correcto.

En USJ hay dos ejemplares determinados con este nombre:

Alajuela, San Ramón. 800 - 1000 m. Luis Acosta s.n. Febrero de 1987, floreció en cultivo en marzo de 1990 (USJ-31985).

Alajuela; Cataratas de San Ramón. 800-1000 m. Luis Acosta s.n. 19/3/1992 (USJ-44878).

"Trichopilia xanthina Mora-Retana \& J. García", nom. herb.

Alajuela; San Ramón. Dora E. Mora s.n. Floreció en el Jardín Botánico Lankester, junio de 1990 (USJ-31981).

Este nombre fue anotado por Dora Emilia en la etiqueta de herbario, pero nunca se publicó. Mario A. Blanco determinó este espécimen en 2000 como T. galeottiana A. Rich. \& Gal.

Trichosalpinx navarrensis (Ames) Mora-Ret. \& J. García, Brenesia 37: 125. 1992.

Basiónimo: Pleurothallis navarrensis Ames, Sched. Orch. 9: 34-35, fig. 5. 1925.

No existe hasta ahora material de esta especie en USJ. En CR sólo hay un ejemplar: Puntarenas; Península de Osa. Aguabuena de Rincón, Fila Casa Loma, siguiendo el antiguo camino a Rancho Quemado. 8' 43'00', N, 83'32' 00', O. 500 m. Gerardo Herrera 4440. 2/10/1990 (CR-210573).

\section{ESPECIES DEDICADAS A DORA EMILIA MORA Y A SU FAMILIA}

1. Epidendrum mora-retanae Hágsater, Icon. Orchid. 2, pars 1: pl. 160. 1993.

Alajuela; Lajas de Alfaro Ruiz. Dora E. Mora s.n. Julio de 1997 (USJ-67287).

Esta especie habita desde Nicaragua hasta
Panamá entre 600 y $1800 \mathrm{~m}$ de altitud. El holotipo es de Panamá; Cuesta de Piedra, Finca El Milagro, 800-1000 m. Leg. Éric Hágsater 6499 \& R.L. Dressler (AMO).

2. Kefersteinia retanae G. Gerlach ex C.O. 
Morales, Brenesia 52: 75. 1999 [2000]

Günter Gerlach (M y Jardín Botánico de Múnich, Alemania) publicó esta especie en Brenesia 41-42:100, 1994, pero faltaba información necesaria para validar el nombre. En Brenesia 52: 75, 1999, se aporta esa información.

San José, Pérez Zeledón, Peña Blanca. 600 m.

Günter Gerlach s.n. Floreció en cultivo en el Jardín Botánico de Múnich en junio de 1992 (Holotipo, USJ-69249).

3. Sobralia doremiliae Dressler, Novon 5(2): 142. 1995.

Cartago; Paraíso, Orosi, Parque Nacional Tapantí.

Dora E. Mora \& R.L. Dressler s.n. 12/11/1993 (Paratipo, USJ-50001).

En el artículo, Dressler explica que prefirió eliminar la a de Dora en doremiliae para evitar una pronunciación errónea. En latín clásico, doraemiliae se pronunciaría "doraimiliai", mientras que en latín vulgar, que es más utilizado por los botánicos, la pronunciación de doremiliae es "doremilie".

4. Stelis morae Luer, Lindleyana 11(2): 103, f. 32. 1996.

El ejemplar tipo (C. Luer 17375) fue recolectado por Dora E. Mora \& R.L. Dressler en San Carlos de Alajuela en enero de 1990. En floración en el Jardín Botánico Lankester el 7/3/1995. La publicación indica que el holotipo está en el Herbario Nacional de Costa Rica (CR), lo cual no es cierto. Un isotipo debiera hallarse en MO. Un solo ejemplar en USJ:

San José: Pérez Zeledón, sin más datos. Leg. W. Fonseca s.n. (F. Pupulin 1710). Floreció en cultivo en San Isidro del General. 21/1/1999. (USJ71130).

5. Telipogon retanarum Dodson \& Escobar, Orquideología 17(2): 114. 1987.

Una de las muchas especies de orquídeas del Parque Nacional Tapantí, en Cartago. Es endémica en Costa Rica y probablemente posee poblaciones muy pequeñas, pues sólo se conocen dos ejemplares tipo de 1984 y, de la misma localidad, dos ejemplares más de 1985, como consta en Orquideología 17(2): 119. 1987.

Cartago; Parque Nacional Tapantí, 1630 m de altitud, ca. a $6 \mathrm{~km}$ de la entrada, $200 \mathrm{~m}$ después de una catarata grande. Rodrigo Escobar, D. Portillo \& M. Retana 3628. 8/8/1985 (USJ27440).

Este ejemplar tiene una etiqueta manuscrita de Dora Emilia, con el \# 3555 de R. Escobar et al. de 1984, que es el tipo de la especie. En marzo de 1992, tanto Dora Emilia como Rodrigo me indicaron que el ejemplar de USJ era un isotipo. Sin embargo, al revisar la publicación en Orquideología y una etiqueta de herbario más una nota que envió Escobar en julio de 2001, es claro que se trata de R. Escobar et al. \# 3628, que no es un tipo. Aun así, es un ejemplar tan valioso como un tipo, pues procede de la localidad típica y hasta ahora es el único de esta especie en los herbarios de Costa Rica.

\section{Publicaciones de Dora Emilia Mora de Retana y sus colegas (En orden cronológico)}

Mora, Dora E. 1971. Desarrollo y crecimiento del ápice vegetativo en Acnistus arborescens. I. Estructura, cambios plastocrónicos y primeros estadios en el desarrollo de la hoja. Rev. Biol. Trop. 19(1,2): 171-189.

Mora, Dora E. 1975. Desarrollo y crecimiento del ápice vegetativo en Acnistus arborescens. II. Desarrollo del procambio y los tejidos vasculares de la hoja y crecimiento de la lámina foliar. Rev. Biol. Trop. 23(2): 155-163.
Mora-Retana, Dora E. (sin año, circa 1983). Gongora. In: Asociación Costarricense de Orquideología (ACO). Orquídeas, su cultivo en Costa Rica. San José, Impresora Delta. p. 26-28.

Rodríguez, R.L., Mora, Dora E., Barahona, María E. \& Williams, N.H. 1986. Géneros de orquídeas de Costa Rica. San José, Edit. Universidad de Costa Rica. 334 p.

Mora Monge, Dora E. \& Valerio G., C. 1988. 
Polinización y producción de frutos en la guaria morada (Cattleya skinneri, Orchidaceae) en Costa Rica. Orquideología 17(3): 242-251.

Mora-Retana, Dora E. \& García-Castro, J. 1990. Nuevas combinaciones de Orchidaceae de Costa Rica. Brenesia 33: 123-126.

Mora, Dora E. 1991. El género Lockhartia Hook. In: Orquídeas Nativas de Colombia 54(2). Sociedad Colombiana de Orquideología.

Mora-Retana, Dora E. \& Atwood, J.T. 1992. Orchids of Costa Rica, part 2. Ic. Pl. Trop. 15, pl. 1401-1500.

Mora-Retana, Dora E. \& García-Castro, J.B. 1992. Nuevas combinaciones en Orchidaceae de Costa Rica (II). Brenesia 37: 125-126.

Mora-Retana, Dora E. \& García-Castro, J.B. 1992. Lista actualizada de las orquídeas de Costa Rica (Orchidaceae). Brenesia 37: 79-124.

Dressler, R.L. \& Mora-Retana, Dora E. 1993. Scaphyglottis geminata, an attractive new species from Costa Rica. Orquídea (Méx.) 13(1-2): 191194.

Dressler, R.L. \& Mora-Retana, Dora E. 1993. Kefersteinia excentrica, a distinctive new species from Costa Rica. Orquídea (Méx.) 13(1-2): 261264.

García-Castro, J.B. Mora-Retana, Dora E. \& Rivas, Marta E. 1993. Lista de orquídeas comunes a Costa Rica y Panamá. Brenesia 39-40: 93-107.

Mora-Retana, Dora E. \& Atwood, J.T. 1993. Orchids of Costa Rica, part 3. Ic. Pl. Trop. 16, pl. 1501-1600.

Mora-Retana, Dora E. \& García-Castro, J.B. 1993. Fernandezia tica Mora-Retana \& J.B. García, una nueva especie orquidácea de Costa Rica. Brenesia 39-40: 163-165.

González, María I., Mora-Retana, Dora E. \& Warner, J. 1994. Crecimiento de Cattleya dowiana (Orchidaceae) en varias condiciones de cultivo. Rev. Biol. Trop., 42 (1/2):73-79.
Pupulin, F. \& Mora-Retana, Dora E. 1994. A revision of the Costa Rican species of Trichocentrum (Orchidaceae). Selbyana 15(2): 87-103.

Mora-Retana, Dora E. \& Warner, J. 1995. La conservación de las orquídeas en el Jardín Botánico Lankester. In: Asociación Costarricense de Orquideología (ACO). Orquídeas de Costa Rica y su cultivo. Vol. I: Cattleya y géneros relacionados. San José, Litografía e Imprenta Lil. p. 54-56.

González L., María I. \& Mora-Retana, Dora E. 1996. El color de las flores en la taxonomía de cuatro especies de Stanhopea (Orchidaceae). Rev. Biol. Trop. 44(2): 525-539.

Mora-Retana, Dora E. \& González L., María I. 1996. Variabilidad floral de cuatro especies de Stanhopea (Orchidaceae). Rev. Biol. Trop. 44(2): 517-523.

Atwood, J.T. \& Mora, Dora E. 1997. Maxillaria cedralensis, a new species from the highlands of Costa Rica. Selbyana 18(1): 31-32.

Mora, Dora E. \& Warner, J. 1997. Orquídeas de Costa Rica. Cartago, Jardín Botánico Lankester, Universidad de Costa Rica. 32 p.

Mora-Retana, Dora E. \& Pupulin, F. 1997. Two new species of Macroclinium (Orchidaceae) from Costa Rica. Selbyana 18(1): 7-10.

Atwood, J.T. \& Mora-Retana, Dora E. 1999. Orchidaceae: Tribe Maxillarieae: Subtribes Maxillariinae and Oncidiinae. In: Burger, W. (ed.). Flora costaricensis. Fieldiana, Bot., n.s. 40: 1-182.

Agradezco sinceramente la colaboración de Robert L. Dressler (FLAS), Éric Hágsater (AMO) y Jorge Warner (Jardín Botánico Lankester) en la preparación de este escrito. 